\title{
Radiative topological biphoton states in modulated qubit arrays
}

\author{
Yongguan Ke, ${ }^{1,2}$ Janet Zhong $\odot,{ }^{2}$ Alexander V. Poshakinskiy, ${ }^{3}$ Yuri S. Kivshar, ${ }^{2,4}$ Alexander N. Poddubny, ${ }^{2,3,4, *}$ \\ and Chaohong Lee ${ }^{1,5, \dagger}$ \\ ${ }^{1}$ Guangdong Provincial Key Laboratory of Quantum Metrology and Sensing \& School of Physics and Astronomy, Sun Yat-Sen University, \\ Zhuhai Campus, Zhuhai 519082, China \\ ${ }^{2}$ Nonlinear Physics Center, Research School of Physics, Australian National University, Canberra ACT 2601, Australia \\ ${ }^{3}$ Ioffe Institute, St. Petersburg 194021, Russia \\ ${ }^{4}$ ITMO University, St. Petersburg 197101, Russia \\ ${ }^{5}$ State Key Laboratory of Optoelectronic Materials and Technologies, Sun Yat-Sen University, Guangzhou Campus, Guangzhou 510275, China
}

(Received 24 February 2020; accepted 13 July 2020; published 4 August 2020)

\begin{abstract}
We study topological properties of bound pairs of photons in spatially modulated qubit arrays (arrays of two-level atoms) coupled to a waveguide. While bound pairs behave like Bloch waves, they are topologically nontrivial in the parameter space formed by the center-of-mass momentum and the modulation phase, where the latter plays the role of a synthetic dimension. In a superlattice where each unit cell contains three two-level atoms (qubits), we calculate the Chern numbers for the bound-state photon bands, which are found to be $(1,-2,1)$. For open boundary conditions, we find exotic topological bound-pair edge states with radiative losses. Unlike the conventional case of the bulk-edge correspondence, these novel edge modes not only exist in gaps separating the bound-pair bands but they also may merge with and penetrate into the bands. By joining two structures with different spatial modulations, we find long-lived interface states which may have applications in storage and quantum information processing.
\end{abstract}

DOI: 10.1103/PhysRevResearch.2.033190

\section{INTRODUCTION}

Topological photonics has attracted a lot of attention recently $[1,2]$. Seminal works on topological photonics have focused on basic topological effects, such as topological edge states [3-5], Floquet topological insulators [6,7], and Weyl and Dirac points $[8,9]$. However, due to their unique optical properties, topological photonic structures can go beyond the straightforward generalizations of topological solid-state systems. In photonic systems, gain and loss can be easily engineered [10], and light-matter interaction can be tailored at will [11-13]. Therefore, there exist multiple non-Hermitian photonic systems which not only support novel topological states [14-17] but may also lead to novel optical devices and unusual applications [18-21].

Radiative topological edge states have already been predicted in linear optical systems [22]. Topological lasers and exciton-polariton topological insulators have also been demonstrated experimentally [18-20]. However, topological photonics is being explored mainly in the linear regime or classical-optics limit which do not take into account any effects of photon-photon interactions.

\footnotetext{
*poddubny@ coherent.ioffe.ru

$\dagger$ lichaoh2@mail.sysu.edu.cn
}

Published by the American Physical Society under the terms of the Creative Commons Attribution 4.0 International license. Further distribution of this work must maintain attribution to the author(s) and the published article's title, journal citation, and DOI.
Strong photon-photon interactions [12,13] are known to appear in photonic waveguides coupled to atoms [23] or arrays of superconducting qubits [24-26]. These quantum waveguides support various exotic correlated states, such as photon bound states [27], novel twilight states [28], and selfinduced localized states [29], which look very promising for storage and processing of quantum information. By arranging the positions of qubits and designing the waveguide structure, the topological edge states have been analyzed in singleexcitation systems [30-33]. However, exotic effects may emerge in interacting topological systems when two or more particles (or quasiparticle excitations) interact [34-39]. Being analytically and numerically more challenging, the study of an interplay between photon interaction, non-Hermiticity, and topology is an exciting novel avenue to be explored in this field.

In this paper we study the topological properties of two excitations in an atomic array with spatial modulation and photon-mediated long-range coupling. Due to the long-range hopping, even though double excitations are forbidden in an individual qubit, there exist two-excitation bound states whose probability amplitudes decay exponentially with the distance between them. By considering an infinite array with unit cell of three two-level atoms (three qubits), we concentrate on the bound-state bands. By varying the phase of spatial modulation and the center-of-mass momentum of two quasiparticle excitations, we observe the emergence of three bound-state bands of nontrivial topological invariants characterized by a set of the Chern numbers $(-1,2,-1)$. For a finite array, we find the radiative two-excitation topological edge state as a subradiant state with decay rate less than that of an individual qubit. In 


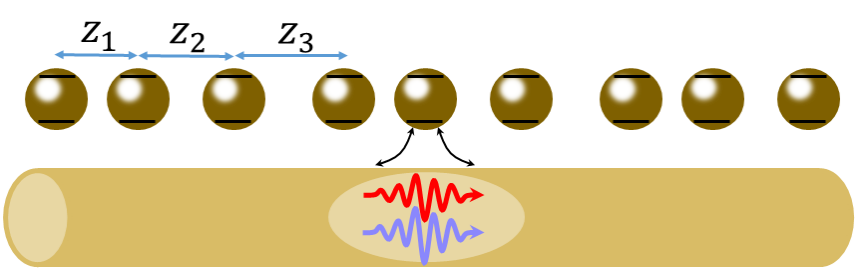

FIG. 1. Schematic of a spatially modulated array of qubits coupled to a waveguide. The modulation period contains three qubits. Coupling between different qubits is mediated by photons propagating along the waveguide.

contrast to the conventional bulk-edge correspondence, these topological bound-edge modes not only exist in the band gaps but also merge and penetrate into the bound-state bands. There also exist long-lived interface states between the two arrays with different spatial modulation phases.

The paper is organized as follows. In Sec. II we introduce the model describing excitations in our qubit array. Next we analyze the band structure and calculate the Chern numbers for the two-excitation bound-state bands (Sec. III). In Sec. IV we calculate the spectral and radiative topological states for a finite structure. In Sec. V we analyze long-lived interface states in a system combining two-qubit arrays with different spatial modulations. Finally, Sec. VI concludes the paper with a brief summary and discussion.

\section{MODEL}

We consider a spatially modulated array of qubits (twolevel atoms) coupled to a waveguide, see Fig. 1. Similar to earlier studies [28,29], we describe the system by the Hamiltonian

$$
\hat{H}=-i \Gamma_{0} \sum_{j, l} b_{j}^{\dagger} b_{l} e^{i \varphi\left|z_{j}-z_{l}\right|}+\frac{\chi}{2} \sum_{j} b_{j}^{\dagger} b_{j}^{\dagger} b_{j} b_{j},
$$

where $\Gamma_{0}$ is the strength of a radiative decay for a single qubit, $b_{j}\left(b_{j}^{\dagger}\right)$ are the annihilation (creation) operators for the bosonic excitations in the $j$ th qubit, $z_{j}$ is the position of the $j$ th qubit, and $\chi$ describes the on-site interaction. The hopping of an excitation from $j$ th to $l$ th qubits is mediated by the waveguide photon which contributes a phase factor depending on the hopping distance. The phase constant $\varphi=\omega_{0} d / c$ depends on the qubit resonant frequency $\omega_{0}$, the light velocity $c$, and the spacing $d$. The qubit positions are modulated according to the relation

$$
z_{j}=j+\delta \cos \left(2 \pi j / \beta+\phi_{0}\right),
$$

where $\beta$ is the spatial period and $\phi_{0}$ is the modulation phase. We choose $\beta=3$, which means that we consider three qubits per unit cell. Such modulation, inspired by the Aubry-AndréHarper model [40], is known to give rise to a nontrivial topology of single-photon bands [41] and radiative edge states [22].

In the limit $\chi \rightarrow \infty$, double excitations in a single qubit are forbidden. Due to the conservation of the excitation number, the Hilbert subspaces for different excitation numbers are decoupled. Below we only consider the subspace of two excitations, where the state can be expanded in the two-excitation basis as $|\psi\rangle=\sum_{j<l} \psi_{j, l} b_{j}^{\dagger} b_{l}^{\dagger}|0\rangle$. For simplicity we denote the basis $b_{j}^{\dagger} b_{l}^{\dagger}|0\rangle \equiv|j ; l\rangle$. Because the qubit array has the period 3 , we can also refer the $(j=3 m+n)$ th qubit with two indices $(m, n)$, where $m$ is the cell index, and $n=1,2,3$ indicates the location in the unit cell.

\section{BAND STRUCTURE AND THE CHERN NUMBER}

For an infinite structure, the total energy is invariant if spatial positions of the two excitations are shifted by a unit cell as a whole. It means that the center-of-mass momentum is a good quantum number according to the many-body Bloch theorem [36-39]. To present the Hamiltonian in a blockdiagonal form with different momenta, we introduce a new basis

$$
|K, \Delta, n\rangle=\sum_{m=1}^{L} e^{i K\left(z_{(m, n)}+z_{(m, n+\Delta)}\right) / 2}|m, n ; m, n+\Delta\rangle .
$$

Here, $K$ is the center-of-mass momentum, $L$ is the truncation number of the unit cells, $\Delta$ is the index difference between the two excitations. Two states may be different even if they have the same value of $\Delta$. Thus we need to distinguish such different states by an additional index $n$, the location of the first excitation in a unit cell. In the new basis, the Hamiltonian elements are given by

$$
\left\langle K^{\prime}, \Delta^{\prime}, n^{\prime}|\hat{H}| K ; \Delta, n\right\rangle=-i \Gamma_{0} \delta_{K, K^{\prime}} M_{\Delta^{\prime}, n^{\prime} ; \Delta, n},
$$

with

$$
\begin{aligned}
M_{\Delta^{\prime}, n^{\prime} ; \Delta, n}= & e^{i \frac{K}{2}\left(z_{n+\Delta}-z_{n+\Delta^{\prime}}\right)} e^{i \varphi\left|z_{n+\Delta}-z_{n+\Delta^{\prime}}\right|} f_{n-n^{\prime}} \\
& +e^{i \frac{K}{2}\left(z_{n}-z_{n+\Delta+\Delta^{\prime}}\right)} e^{i \varphi\left|z_{n}-z_{n+\Delta+\Delta^{\prime}}\right|} f_{n+\Delta-n^{\prime}} \\
& +e^{i \frac{K}{2}\left(z_{n+\Delta}-z_{n-\Delta^{\prime}}\right)} e^{i \varphi\left|z_{n+\Delta}-z_{n-\Delta^{\prime}}\right|} f_{n^{\prime}+\Delta^{\prime}-n} \\
& +e^{i \frac{K}{2}\left(z_{n}-z_{n+\Delta-\Delta^{\prime}}\right)} e^{i \varphi\left|z_{n}-z_{n+\Delta-\Delta^{\prime}}\right|} f_{n+\Delta-\Delta^{\prime}-n^{\prime}}
\end{aligned}
$$

where

$$
f_{x}=\left\{\begin{array}{l}
1, \text { if } \quad \bmod (x, 3)=0 ; \\
0, \text { if } \bmod (x, 3) \neq 0 .
\end{array}\right.
$$

The Hamiltonian elements are nonzero only when $K=K^{\prime}$, in other words, there is no coupling between subspaces with different center-of-mass momenta. By solving the eigenvalue problem $-i \Gamma_{0} \hat{M}\left|u^{(m)}(K)\right\rangle=2 E_{K, m}\left|u^{(m)}(K)\right\rangle$, we can obtain the energy bands depending on the center-of-mass momentum, see Fig. 2(a) and its zoom-in (b). Since the hopping terms are non-Hermitian, the eigenvalues are complex.

To characterize the bound states, we calculate the probability of finding two excitations within a certain short distance $\Delta_{0}$,

$$
P_{m, \Delta_{0}}(K)=\sum_{1 \leqslant n \leqslant n_{0} ; 1 \leqslant \Delta \leqslant \Delta_{0}}\left|u_{\Delta, n}^{(m)}(K)\right|^{2} .
$$

Here, $u_{\Delta, n}^{(m)}(K)$ is the amplitude of the $m$ th eigenstate $\left|u^{(m)}(K)\right\rangle$. The colors in Figs. 2(a) and 2(b) indicate the bounded probability $P_{m, \Delta_{0}}$. Here $n_{0}=3$ is the spatial modulation period, and the other parameters are chosen as $\Gamma_{0}=1, \delta=0.1, \varphi=0.3$, $\phi_{0}=0$, and $\Delta_{0}=5$ (actually, $\Delta_{0}$ can be chosen as another value $\lesssim 10$ which does not affect the features of results). We find that there exist three bound-state bands merging into 

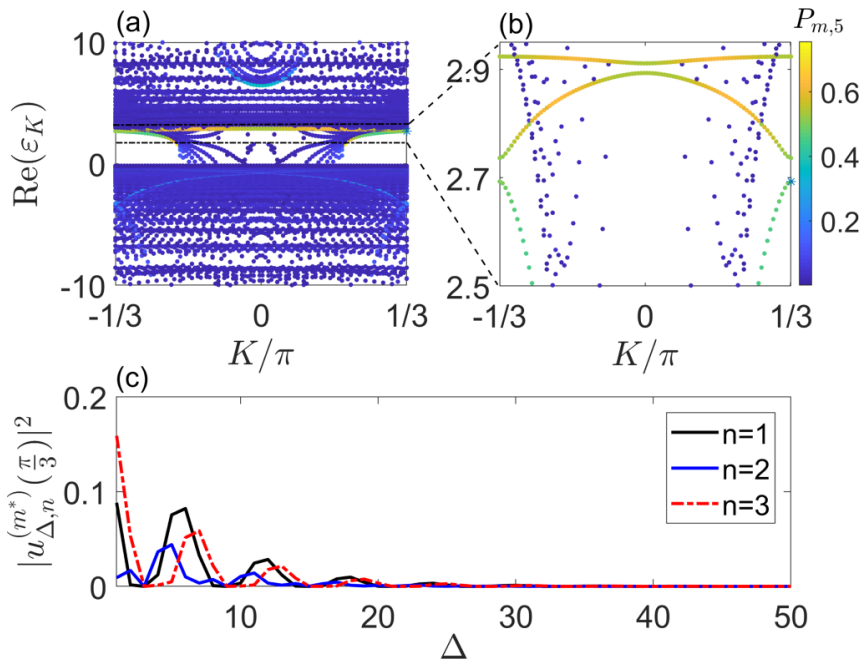

FIG. 2. (a) The band structure vs the center-of-mass momentum for two excitations. The colors denote the probability to find the two excitations within a certain short distance $\Delta_{0}=5$. (b) The zoom-in of (a) showing the position of considered three bound-state bands. (c) The probability as a function of the distance $\Delta$ between two excitations. The parameters are chosen as $\Gamma_{0}=1, \delta=0.1, \varphi=0.3$, $L=99$, and $\phi_{0}=0$.

the scattering bands. The decay rates of these bound states decrease with the qubit number. The origin of these three bound-state bands is similar to the formation of conventional energy bands. For a simple periodic array, a bound-state band is folded in the reduced Brillouin zone $(-\pi / 3 \leqslant K \leqslant \pi / 3)$. The gaps between bands are open at the degenerate points as the modulation strength is increased from 0 .

We show the probability amplitude $\left|u_{\Delta, n}^{\left(m^{*}\right)}(K)\right|^{2}$ of the bound state with $K=\pi / 3$ as a function of the distance between two excitations, see Fig. 2(c). $n=1,2,3$ indicates the position of the first qubit in a unit cell. The corresponding energy is marked as $*$ in Fig. 2(a). It is clear that the bound state exponentially decays with the relative distance $\Delta$, independent of the position of the qubit in a unit cell. Apart from the bound states, there are many scattering states, which can be viewed as two independent free excitations. The wave function of the scattering state can be decomposed as the product of two single-excitation Bloch functions. If we denote the eigenenergy of the single-excitation Bloch state as $\varepsilon_{k}$, then the energy for the scattering state is approximately given by

$$
E_{K} \approx \frac{\varepsilon_{(K+\kappa) / 2}+\varepsilon_{(K-\kappa) / 2}}{2}
$$

where $\kappa$ is the momentum associated with the relative motion of the two excitations. Since the two excitations are nearly free, the momenta for the two excitations $-\pi / 3 \leqslant$ $(K \pm \kappa) / 2 \leqslant \pi / 3$ are independent, and hence $-2 \pi / 3 \leqslant \kappa \leqslant$ $2 \pi / 3$.

Chern number is a well-known topological invariant which characterizes the band topology in the two-dimensional parameter space. In previous works [36-38], the Chern number is extended to characterize the strongly correlated topological states in two-particle systems. In particular, it has been justified that the Chern number of a two-particle bound-state

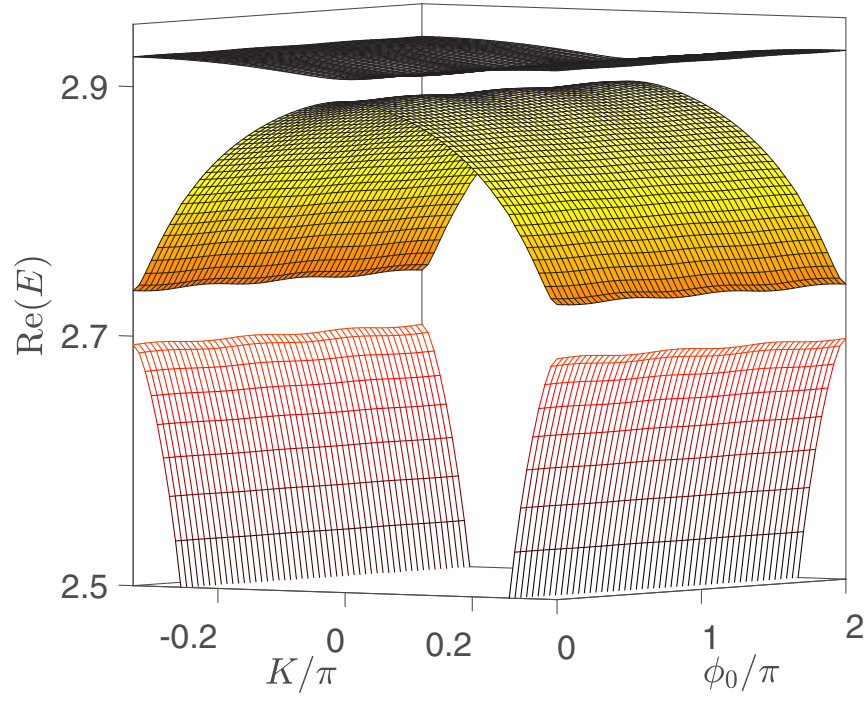

FIG. 3. The bound-state bands vs the center-of-mass momentum $K$ and the modulation phase $\phi_{0}$. The eigenenergy $E$ is in the unit of $\Gamma_{0}$. The parameters are chosen as $\delta=0.1 \Gamma_{0}, L=70$, and $\varphi=0.3$.

band can be used for explaining the Thouless pumping of two interacting bosons [36]. To reveal the topological nature of the bound-state bands, we calculate the Chern number for the bound-state bands in the $\left(K, \phi_{0}\right)$ plane. The Chern number is defined as

$$
C_{m}=\frac{1}{2 \pi} \int_{-\pi / 3}^{\pi / 3} d K \int_{0}^{2 \pi} d \phi_{0} \mathcal{F}_{m}\left(K, \phi_{0}\right)
$$

where the Berry curvature is $\mathcal{F}_{m}=i\left(\left\langle\partial_{\phi_{0}} u^{(m)} \mid \partial_{K} u^{(m)}\right\rangle-\right.$ $\left.\left\langle\partial_{K} u^{(m)} \mid \partial_{\phi_{0}} u^{(m)}\right\rangle\right)$. Because the energies of the bound-state bands merge into those of the scattering-state bands, we need to distinguish bound-state bands from the scatteringstate ones. Here we pick out the bound-state bands if the bounded probability $P_{m, 5}$ is greater than a threshold, $P_{m, 5}>$ $P_{t h}$. The threshold value $P_{t h}=0.25$ is selected by numerical experiments so that it can distinguish between the smooth and continuous surface of bound-state bands. The bound-state bands depending on $K$ and $\phi_{0}$ are shown in Fig. 3. The top two bands are continuous in the $\left(K, \phi_{0}\right)$ plane, and thus we can directly apply Eq. (9) to calculate the Chern numbers. On the other hand, the lowest band has two disconnected two branches which have linear dispersion when $K$ approaches to $\pm K_{0} \approx \pm 0.19 \pi$. However, the major contributions for Chern number come from the Berry curvature around the band edge $K= \pm \pi / 3$. Thus we sum the Berry curvature of the two branches to get the Chern number. The Chern numbers for the three bands from bottom to the top are $(-0.9318,1.9505,-0.9894)$ for the cell number $L=70$, which tend to the ideal integers $(-1,2,-1)$ as $L$ increases. Similarly, in principle we can also calculate the Chern numbers for scattering states when the scattering-state bands are well distinguished, which originate from the single-particle topology. 


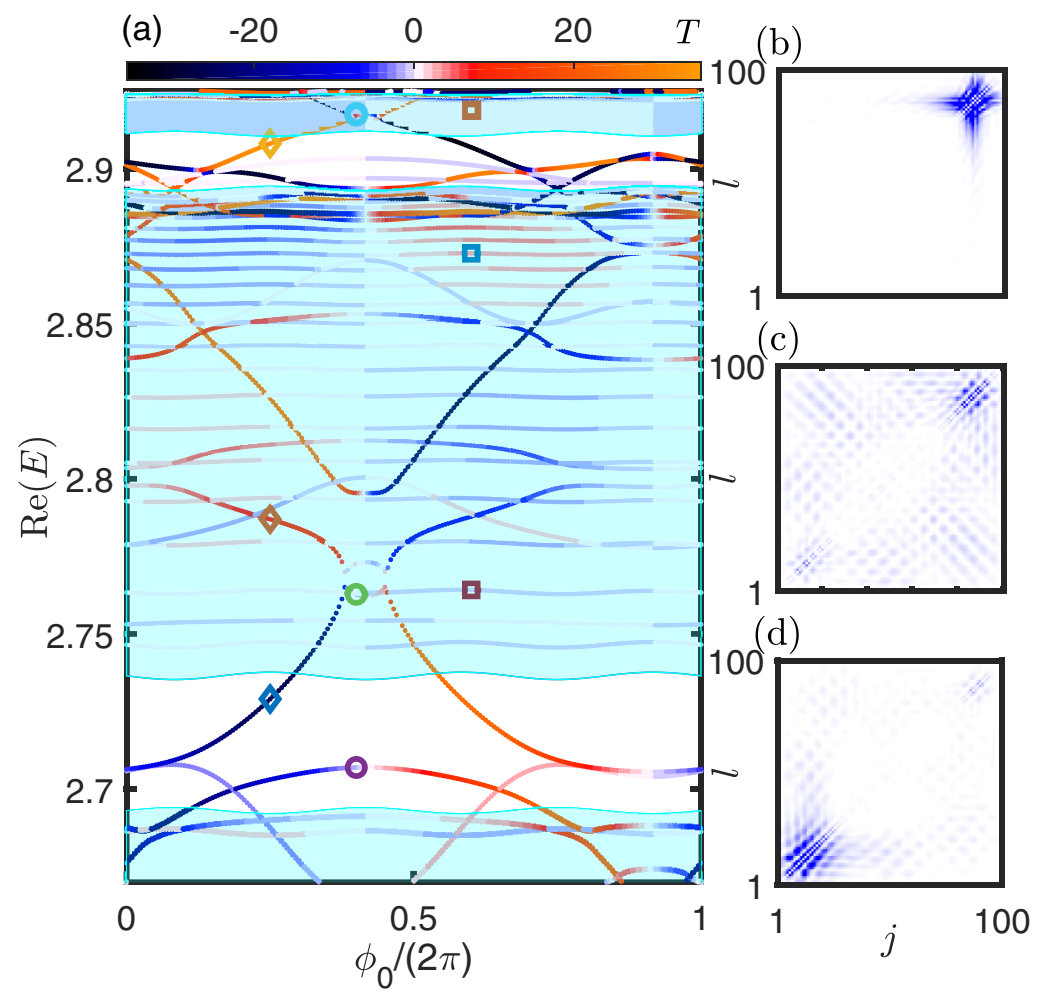

(e)

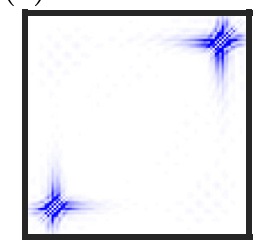

(f)

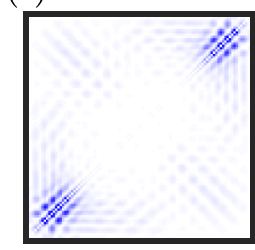

(g)

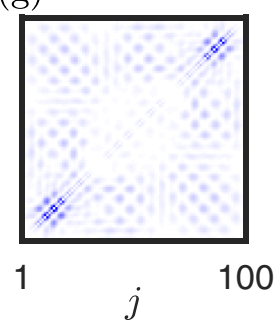

(h)

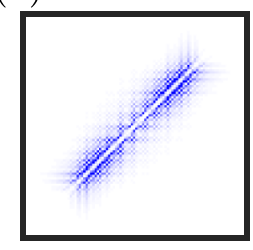

(i)

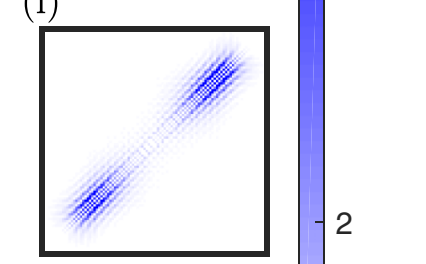

(j)

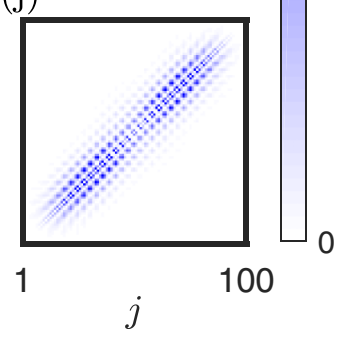

FIG. 4. Energy spectrum and topological states in a finite structure. (a) Energy spectrum vs the modulation phase. The colors in (a) denote the mean center-of-mass position. (b)-(d) Eigenstates at $\phi_{0}=2 \pi / 5$ with approximate energies $E=2.91-0.04 i, E=2.79-0.06 i$, and $E=2.73-0.09 i$, marked by diamonds from top to bottom in (a), respectively. (e)-(f) Eigenstates at $\phi_{0}=4 \pi / 5$ with approximate energies $E=2.92-0.05 i, E=2.76-0.07 i$, and $E=2.71-0.06 i$, marked by circles from top to bottom in (a), respectively. (h)-(j) Eigenstates at $\phi_{0}=6 \pi / 5$ with approximate energies $E=2.92-0.001 i, E=2.87-0.008 i$, and $E=2.76-0.007 i$, marked by squares from top to bottom in (a), respectively. The colors in (b)-(j) denote the probability $\left|\psi_{j, l}\right|^{2}$.

\section{RADIATIVE TOPOLOGICAL STATES}

In this section, we proceed to the spectrum and eigenstates in a finite and modulated array. There is always radiative loss for a finite array with qubit-photon coupling, that is, the excitations will be transferred into photons and escape from the array into the waveguide. The radiative decay rate is determined by the imaginary part of the eigenvalue, $\Gamma=$ $-\operatorname{Im}(\varepsilon)[28]$. Thus all of the eigenstates in a finite array have radiative losses.

We now study the energy spectrum as a function of the modulation phase, see Fig. 4. The parameters are chosen as $\Gamma_{0}=1, \varphi=0.3, \delta=0.1$ and the number of qubits is $N=$ 100. We focus on the energy spectrum around the bound-state bands which are shaded in cyan and check the bulk-edge correspondence. To distinguish the edge and scattering states, we calculate the mean center-of-mass position of the two excitations,

$$
T=\sum_{j, l} \frac{1}{2}[j+l-(N+1)]\left|\psi_{j, l}\right|^{2},
$$

where the center position is shifted to the origin point. For $N=100, T$ takes the maximum and minimum values $\pm(N / 2-2)= \pm 48$ if the two excitations are located at the leftmost and the rightmost edges, respectively. On the other hand, $T$ is close to zero if the two excitations are in a scattering state or in an equal superposition of left and right edge states.
It means that $T$ can be used to select the states localized at one edge. The colors in Fig. 4(a) denote the mean center-of-mass position of the corresponding eigenstates where black/dark blue and red signify left and right localized edge states, respectively. In the band gaps, we can find black and red curves entering the bound-state bands. There also exist dark blue and red curves entirely merging in the bound-states bands. To get a relatively complete information about the bound states, we color the spectrum with the bound probability, $P_{\text {bound }}=\sum_{|j, l| \leqslant \Delta_{0}}\left|\psi_{j, l}\right|^{2}$; see Fig. 5. Larger bound probability indicates that the two excitations are strongly bounded with relative distance less than $\Delta_{0}$. Here, we choose $\Delta_{0}=10$. It is clear that some scattering states also exist in the continuum of bound-state bands, consistent with the result of the infinite structure in Fig. 2(a).

To study the correlation properties, we calculate the joint probability distribution $\left|\psi_{j, l}\right|^{2}$ for one excitation at the $j$ th qubit and the other one at the $l$ th qubit. For $\phi_{0} /(2 \pi)=1 / 5$, we choose three typical states marked by diamonds from the bottom to the top in Fig. 4(a), with the larger absolute values of $T$. The top diamond in the second gap represents the state when two excitations are bounded within short distance and close to the right edge, see Fig. 4(b). It also has the crossing feature, which may be affected by the self-localized states with close energies [29]. Such state can be termed as a bound-edge state. The bottom diamond represents a boundedge state localized at the left edge, see Fig. 4(d). The middle 


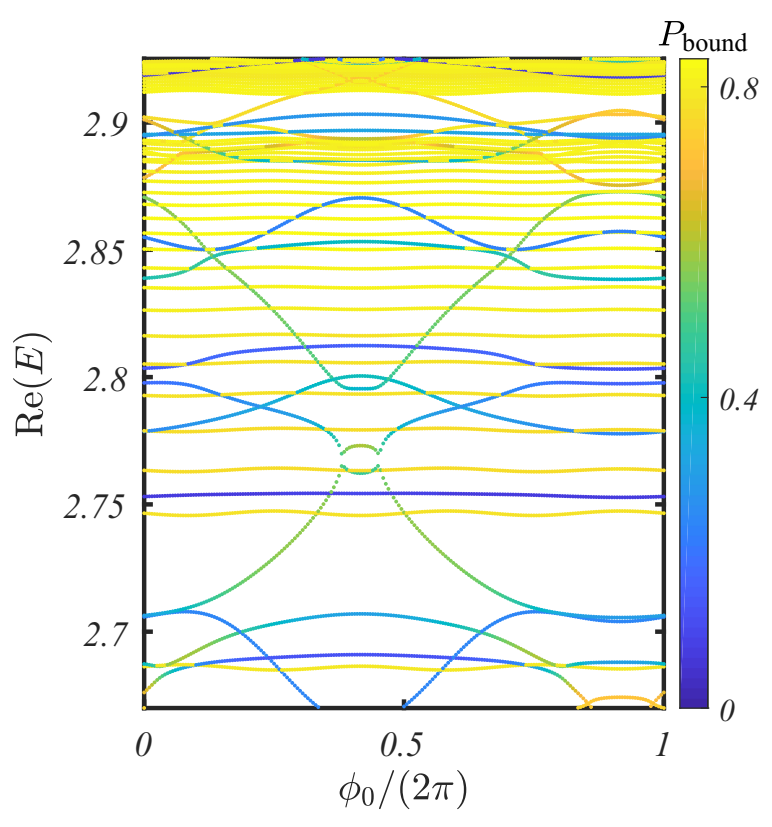

FIG. 5. Spectrum colored by the bound probability $P_{\text {bound }}=$ $\sum_{|j, l| \leqslant 10}\left|\psi_{j, l}\right|^{2}$. The parameters are the same as those in Fig. 4(a).

diamond represents the bound-edge state slightly mixed with the scattering states, see Fig. 4(c). This coexistence of boundedge and scattering states in a single eigenstate confirms again that the bound-edge states not only exist in the band gaps but also merge and penetrate into the bound-state bands.

Next we choose three states for the phase $\phi_{0} /(2 \pi)$ close to $2 / 5$, marked by circles from top to the bottom, which connect the dark blue and red curves, see Figs. 4(e)-4(g). These states are in the superposition of bound states localized at the left and right edges. These symmetric or asymmetric bound-edge states have a decay rate in the order of $\varphi^{2} \Gamma_{0}$. It is interesting that the phase values $\phi_{0} /(2 \pi)=2 / 5+1 / 60$ and $\phi_{0} /(2 \pi)=2 / 5+1 / 60+1 / 2$ correspond to the critical points where all the states are symmetrically distributed with respect to the array center. This is because the system preserves inversion symmetry at these critical points, that is, the energy is unchanged when changing the position of two excitations at $(j, l)$ to $(N+1-l, N+1-j)$. The critical point is obtained by solving $\cos \left[2 \pi / 3(j+1)+\phi_{0}\right]-\cos \left(2 \pi / 3 j+\phi_{0}\right)$ $=\cos \left[2 \pi / 3(N+1-j)+\phi_{0}\right]-\cos \left[2 \pi / 3(N-j)+\phi_{0}\right]$,

that is, $\cos \left(\phi_{0}-2 \pi / 3\right)+\cos \left(\phi_{0}\right)=2 \cos \left(\phi_{0}+2 \pi / 3\right)$ for $N=100$. The critical points are shifted by multiples of $2 \pi / 3$ as the number of qubits changes. Away from such critical points, the two-excitation states tend to either the left or the right part of the array.

For $\phi_{0} /(2 \pi)=3 / 5$, we focus on the three scattering states marked as squares in the bulk bands, see Figs. 4(h)-4(j). The probability distributions are concentrated along the diagonal line $l=j$, which is a clear signature of the bound states. The bound states in the bulk have a decay rate smaller than that of the bound-edge states by an order of magnitude. The intuitive explanation is that the radiative loss happens at the edge qubits, and the bound states in the bulk have less occupation at the edge than that of the bound-edge states [42]. These bound

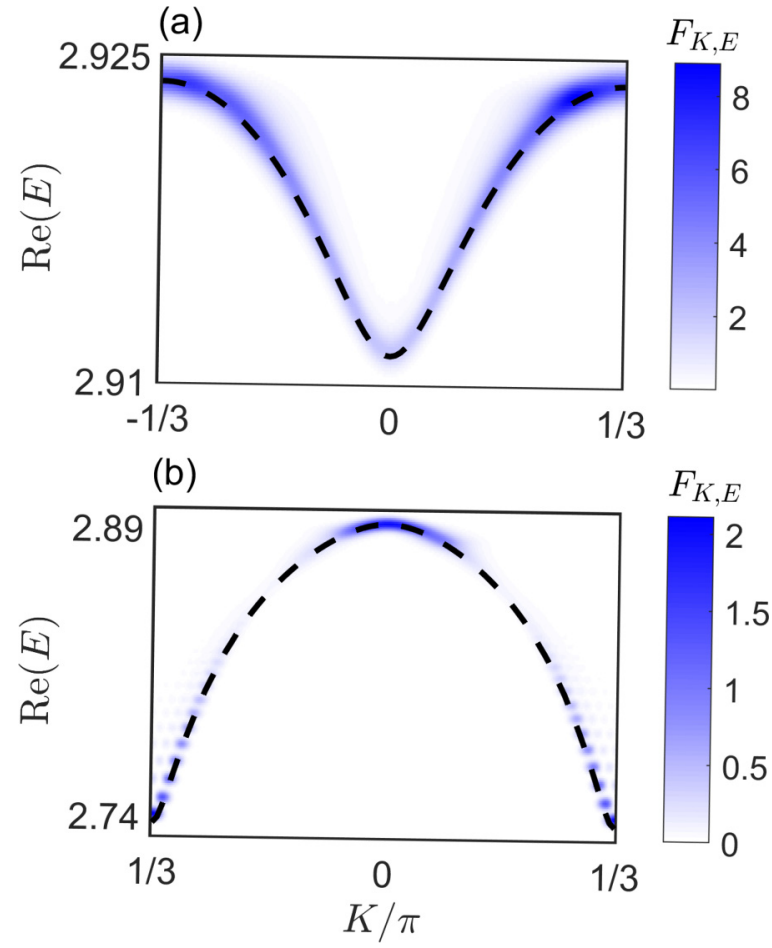

FIG. 6. Density distribution of bound states in the $(K, E)$ plane around (a) the third bound-state band and (b) the second bound-state band. The dashed lines indicate the third and second bound-state bands. The parameters are chosen the same as those in Fig. 2.

states can be classified as subradiant states, which have the radiative lifetime larger than that of the single qubit.

The center-of-mass motion of the bound states behaves as a Bloch wave with momentum $K$. To extract the center-of-mass momentum, we make a Fourier transformation of the bound states:

$$
\Psi_{s, n}^{(v)}(K)=\sum_{m=0}^{M-1} \psi_{3 m+n-s, 3 m+n}^{(v)} e^{i K\left(z_{3 m+n}+z_{3 m+n-s}\right) / 2},
$$

where $v$ refers to the $v$ th eigenstates, $s$ restricts the distance between two excitations, and $n$ is the position index in a unit cell. Since we know the center-of-mass momentum and the eigenvalues, we can reconstruct the dispersion relation by calculating the wave-vector-resolved density of states [43],

$$
F(E, K)=\sum_{v} e^{-\frac{\left|E-\operatorname{Re}\left(E_{v}\right)\right|^{2}}{2 \sigma^{2}}} \sum_{s, n}\left|\Psi_{s, n}^{(v)}(K)\right|^{2},
$$

where the Gaussian broadening $\sigma$ is introduced for better visualization. Figures 6(a) and 6(b) show the density of states for the bound states in the second and third bound-state bands, respectively. The parameters are chosen as $\Gamma_{0}=1, \varphi=0.3$, $\delta=0.1, \phi_{0}=0, s=10, M=50$, and $\sigma=1 \times 10^{-3}$. The value of $\sigma$ can be chosen differently, since it only affects the width of density distribution. The dashed lines in Figs. 6(a) and 6(b) show the second and third bound-state center-ofmass dispersion, respectively, and well match the density of states, Eq. (12). This means that our calculations in the finite structure and in the infinite structure are consistent. 


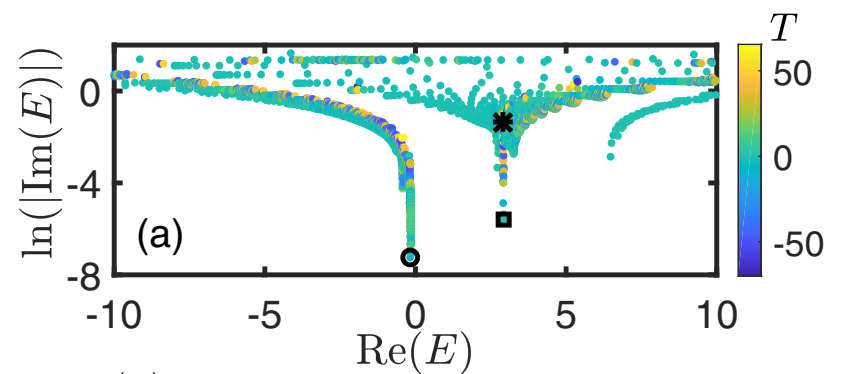

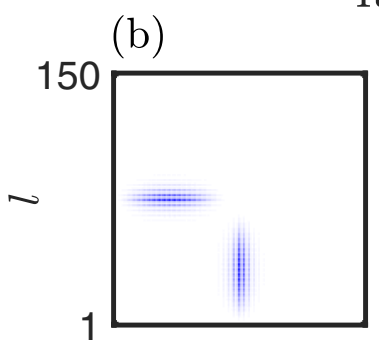

(d)
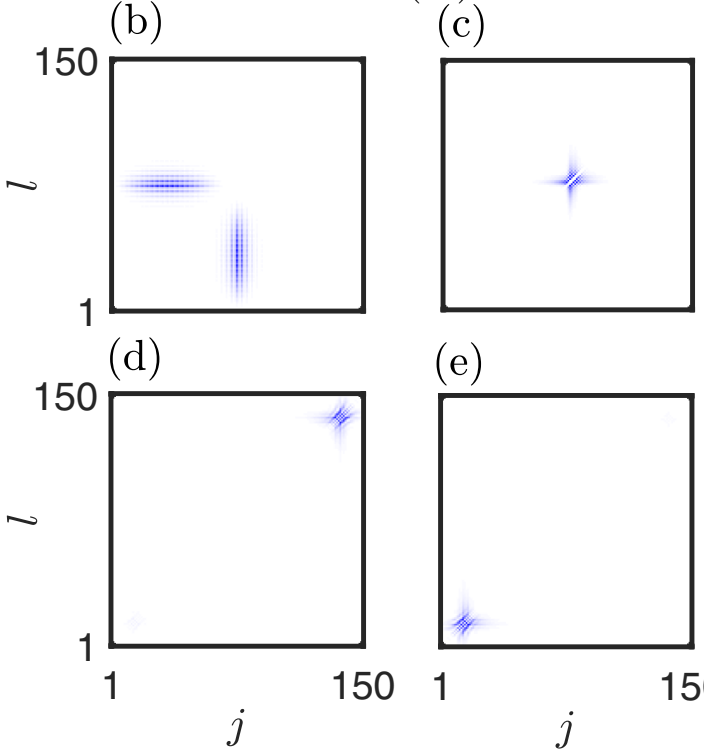

(e)

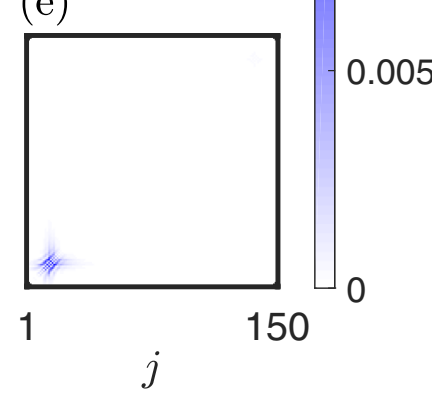

FIG. 7. (a) The eigenvalue distribution in the complex plane. The colors denote the mean center-of-mass position of the two excitations. (b)-(e) The probability distribution for two excited states with approximate energies $E=-0.18-5.7 \times 10^{-8} i, E=2.93-$ $2.5 \times 10^{-6} i, E=2.90-0.043 i$, and $E=2.90-0.045 i$, marked by $\circ, \square, \times$, and + in (a), respectively.

\section{LONG-LIVED INTERFACE STATES}

According to our calculations, the bound-edge states have the finite radiative decay rate on the order of $\sim \varphi^{2} \Gamma_{0}$. This is because these states appear at an interface between the qubit array and free space, where the radiative loss becomes significant [42]. However, if we connect two-qubit arrays with different modulation phases, there should be edge states with longer lifetime at the interface located in the bulk of the array. The qubit at the interface plays the role of a defect which can trap the excitations for a long time. To verify this argument, the positions of qubits are arranged as

$$
z_{j}=\left\{\begin{array}{l}
j+\delta \cos (2 \pi j / 3), j \leqslant N / 2, \\
j+\delta \cos (2 \pi j / 3+\pi), j>N / 2 .
\end{array}\right.
$$

There is an interface between $N / 2$ and $N / 2+1$ qubits.

We calculate the energy distribution in the complex plane, as shown in Fig. 7 for the parameters $\delta=0.1, \varphi=0.3, \Gamma_{0}=$ 1 , and $N=150$. The colors mark the mean center-of-mass position of the corresponding eigenstates. We are interested in the most subradiant state with the energy marked as a circle in Fig. 7(a), and its probability distribution is shown in Fig. 7(b). Such a state is approximated by an antisymmetric combination [44] of the single-excitation interface state and the most subradiant single-excitation state at the left part of the qubit array, as discussed in Appendix. We also find a kind of crosslike interface state, which is a mixture of a bound state and a self-localized state with the energy marked as a square, see Fig. 7(c). Such interface states are induced by the interplay between topology, interaction, and non-Hermitian coupling. These two kinds of interface states have much smaller decay rates $\left(\Gamma<10^{-6} \Gamma_{0}\right)$ than those of the bound-edge states $\left(\Gamma \sim 10^{-2} \Gamma_{0}\right)$ in Figs. 7(d) and 7(e). Importantly, the decay rate for the interface states decreases as the qubit number increases. However, for the bound edge states (marked as "+" and " $x ")$, either localized at the left or right edges, the decay rates are in the order of $10^{-2} \Gamma_{0}$, which are in the same order as those in Fig. 4, even with the increase of the system size.

\section{SUMMARY AND DISCUSSION}

We have studied topological properties of bound photons propagating in spatially modulated atomic arrays coupled to a waveguide. We have calculated the center-of-mass bands and characterized the topology of bound-state bands with the Chern numbers. In contrast to many conventional systems realizing the bulk-edge correspondence, our system supports radiative bound-edge states which can be associated with both the band gaps and also bulk bands. When the modulation phase is modified and passes through the critical points, the edge states localized in the left part of the array transform to those localized in its right part, or vice versa. When two arrays of qubits with different spatial modulations are connected, they can support long-lived interface topological states.

Previously, the breakdown of conventional bulk-edge correspondence has been discussed in either non-Hermitian or interacting systems. For noninteracting non-Hermitian systems, the generalized bulk-edge correspondence has been suggested by employing non-Bloch topological invariants [15] or biorthogonal polarization [45]. For interacting Hermitian systems, the breakdown of bulk-edge correspondence may be explained by the virtual defect induced by particle-particle interactions. However, both non-Hermiticity and interaction coexist in our systems, making this type of problem much more complicated [46], so further study is required to uncover the principle of bulk-edge correspondence in our system.

Recently, topological features have been employed to improve the optical properties and control the optical response. Out-of-plane-scattering losses, a hindrance for high- $Q$ resonators, can be suppressed by the topological nature of the bound state in continuum [47]. Our previous study has revealed that double-excited subradiant states enable a longer time for light-matter interaction, and they can enhance the inelastic scattering of photon pairs [28]. An interesting avenue for future exploration of these kinds of systems is to study whether the topological interface states can be used to manipulate the correlation and entanglement of photon pairs.

\section{ACKNOWLEDGMENTS}

This work is supported by the Key Area Research and Development Program of GuangDong Province under Grant No. 2019B030330001, the National Natural Science 

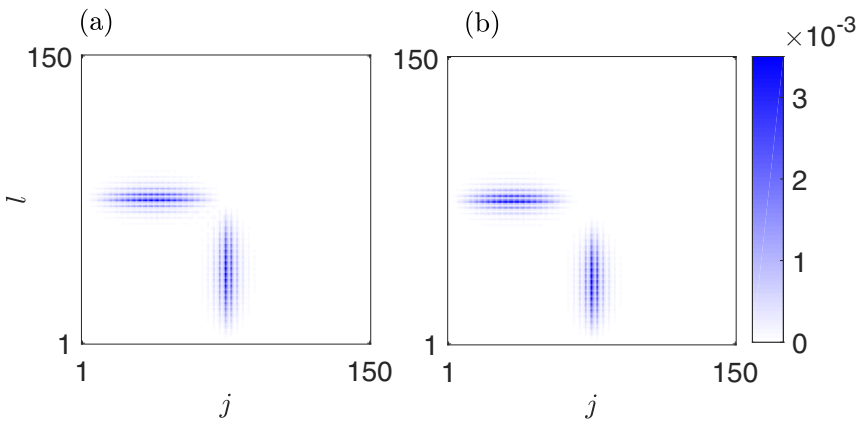

FIG. 8. Probability distribution $\left|\psi_{j, l}\right|^{2}$ as a function of positions $j$ and $l$ for (a) the fermionlike ansatz and (b) the most subradiant interface state.

Foundation of China (NNSFC) under Grants No. 11874434 and No.11574405, and the Science and Technology Program of Guangzhou (China) under Grant No. 201904020024. Y.K. is partially supported by the Office of China Postdoctoral Council (Grant No. 20180052), the National Natural Science Foundation of China (Grant No. 11904419), and the Australian Research Council (DP200101168). J.Z. is supported by the Australian Government Research Training Program (RTP) Scholarship. A.V.P. acknowledges a support from the Russian Science Foundation (Project No. 19-72-00080). A.N.P. has been supported by the Russian President Grant No. MD243.2020.2. Y.S.K. is supported by the Australian Research Council.

\section{APPENDIX: APPROXIMATION OF THE INTERFACE STATE}

Here we show how the interface state can be approximated by an antisymmetric combination of the single-excitation interface state $\left|\psi_{\text {int }}^{(1)}\right\rangle$ and the most subradiant state $\left|\psi_{L}^{(1)}\right\rangle$ at the left part of the array. By diagonalizing the Hamiltonian (1) with $z_{j}$ arranged as Eq. (13) in the single-excitation subspace, we obtain the interface state $\left|\psi_{\text {int }}^{(1)}\right\rangle=\sum_{j} c_{j}|j\rangle$, with the longest lifetime. By diagonalizing the Hamiltonian (1) with $z_{j}=j+\delta \cos (2 \pi j / 3)[j \leqslant N / 2]$ in the singleexcitation subspace, we can also obtain the most subradiant single-excitation state, $\left|\psi_{L}^{(1)}\right\rangle=\sum_{j} f_{j}|j\rangle$ with $f_{j>N / 2}=0$. The ansatz of the most subradiant interface state $\left|\psi_{i n t}^{(2)}\right\rangle=$ $\sum_{j, l} \psi_{j, l}|j, l\rangle$ has the antisymmetric form [44]

$$
\psi_{j, l}= \begin{cases}c_{j} f_{l}-f_{l} c_{j}, & (j \geqslant l) \\ -\left(c_{j} f_{l}-f_{l} c_{j}\right), & (j \leqslant l) .\end{cases}
$$

In Fig. 8 we show the probability distributions $\left|\psi_{j, l}\right|^{2}$ as functions of the positions $j$ and $l$ for (a) fermionlike ansatz and (b) most subradiant interface state. The parameters are chosen the same as those in Fig. 7. It is clear that the fermion ansatz [44] well describes the major features of the most subradiant interface state of two excitations.
[1] L. Lu, J. D Joannopoulos, and M. Soljačić, Topological photonics, Nat. Photonics 8, 821 (2014).

[2] T. Ozawa, H. M. Price, A. Amo, N. Goldman, M. Hafezi, L. Lu, M. C. Rechtsman, D. Schuster, J. Simon, O. Zilberberg, and I. Carusotto, Topological photonics, Rev. Mod. Phys. 91, 015006 (2019).

[3] Z. Wang, Y. Chong, J. D. Joannopoulos, and M. Soljačić, Observation of unidirectional backscattering-immune topological electromagnetic states, Nature (London) 461, 772 (2009).

[4] M. Hafezi, E. A. Demler, M. D. Lukin, and J. M. Taylor, Robust optical delay lines with topological protection, Nat. Phys. 7, 907 (2011).

[5] M. Hafezi, S. Mittal, J. Fan, A. Migdall, and J. M. Taylor, Imaging topological edge states in silicon photonics, Nat. Photonics 7, 1001 (2013).

[6] M. C. Rechtsman, J. M. Zeuner, Y. Plotnik, Y. Lumer, D. Podolsky, F. Dreisow, S. Nolte, M. Segev, and A. Szameit, Photonic Floquet topological insulators, Nature (London) 496, 196 (2013).

[7] L. J. Maczewsky, J. M. Zeuner, S. Nolte, and A. Szameit, Observation of photonic anomalous Floquet topological insulators, Nat. Commun. 8, 13756 (2017).

[8] L. Lu, Z. Wang, D. Ye, L. Ran, L. Fu, J. D. Joannopoulos, and M. Soljačić, Experimental observation of Weyl points, Science 349, 622 (2015).

[9] L. Lu, C. Fang, L. Fu, S. G. Johnson, J. D. Joannopoulos, and M. Soljačić, Symmetry-protected topological photonic crystal in three dimensions, Nat. Phys. 12, 337 (2016).
[10] S. V Suchkov, A. A. Sukhorukov, J. Huang, S. V. Dmitriev, C. Lee, and Y. S. Kivshar, Nonlinear switching and solitons in PT-symmetric photonic systems, Laser Photonics Rev. 10, 177 (2016).

[11] K. E. Dorfman, F. Schlawin, and S. Mukamel, Nonlinear optical signals and spectroscopy with quantum light, Rev. Mod. Phys. 88, 045008 (2016).

[12] D. Roy, C. M. Wilson, and O. Firstenberg, Colloquium: Strongly interacting photons in one-dimensional continuum, Rev. Mod. Phys. 89, 021001 (2017).

[13] D. E. Chang, J. S. Douglas, A. González-Tudela, C.-L. Hung, and H. J. Kimble, Colloquium: Quantum matter built from nanoscopic lattices of atoms and photons, Rev. Mod. Phys. 90, 031002 (2018).

[14] T. E. Lee, Anomalous Edge State in a non-Hermitian Lattice, Phys. Rev. Lett. 116, 133903 (2016).

[15] S. Yao and Z. Wang, Edge States and Topological Invariants of non-Hermitian Systems, Phys. Rev. Lett. 121, 086803 (2018).

[16] Z. Gong, Y. Ashida, K. Kawabata, K. Takasan, S. Higashikawa, and M. Ueda, Topological Phases of Non-Hermitian Systems, Phys. Rev. X 8, 031079 (2018).

[17] V. M. Martinez Alvarez, J. E. Barrios Vargas, M. Berdakin, and L. E. F. Foa Torres, Topological states of non-Hermitian systems, Eur. Phys. J. Spec. Top. 227, 1295 (2018).

[18] M. A. Bandres, S. Wittek, G. Harari, M. Parto, J. Ren, M. Segev, D. N. Christodoulides, and M. Khajavikhan, Topological insulator laser: Experiments, Science 359, eaar4005 (2018). 
[19] G. Harari, M. A. Bandres, Y. Lumer, M. C. Rechtsman, Y. D. Chong, M. Khajavikhan, D. N. Christodoulides, and M. Segev, Topological insulator laser: Theory, Science 359, eaar4003 (2018).

[20] S. Klembt, T. H. Harder, O. Egorov, K. Winkler, R. Ge, M. A. Bandres, M. Emmerling, L. Worschech, T. C. H. Liew, M. Segev et al., Exciton-polariton topological insulator, Nature (London) 562, 552 (2018).

[21] S. Kruk, A. Poddubny, D. Smirnova, L. Wang, A. Slobozhanyuk, A. Shorokhov, I. Kravchenko, B. Luther-Davies, and Y. Kivshar, Nonlinear light generation in topological nanostructures, Nat. Nanotechnol. 14, 126 (2019).

[22] A. V. Poshakinskiy, A. N. Poddubny, L. Pilozzi, and E. L. Ivchenko, Radiative Topological States in Resonant Photonic Crystals, Phys. Rev. Lett. 112, 107403 (2014).

[23] N. V. Corzo, J. Raskop, A. Chandra, A. S. Sheremet, B. Gouraud, and J. Laurat, Waveguide-coupled single collective excitation of atomic arrays, Nature (London) 566, 359 (2019).

[24] A. F. van Loo, A. Fedorov, K. Lalumiere, B. C. Sanders, A. Blais, and A. Wallraff, Photon-mediated interactions between distant artificial atoms, Science 342, 1494 (2013).

[25] M. Mirhosseini, E. Kim, X. Zhang, A. Sipahigil, P. B. Dieterle, A. J. Keller, A. Asenjo-Garcia, D. E. Chang, and O. Painter, Cavity quantum electrodynamics with atom-like mirrors, Nature (London) 569, 692 (2019).

[26] Z. Wang, H. Li, W. Feng, X. Song, C. Song, W. Liu, Q. Guo, X. Zhang, H. Dong, D. Zheng, H. Wang, and D.-W. Wang, Controllable Switching Between Superradiant and Subradiant States in a 10-Qubit Superconducting Circuit, Phys. Rev. Lett. 124, 013601 (2020).

[27] Y.-X. Zhang, C. Yu, and K. Mølmer, Subradiant bound dimer excited states of emitter chains coupled to a one dimensional waveguide, Phys. Rev. Res. 2, 013173 (2020).

[28] Y. Ke, A. V. Poshakinskiy, C. Lee, Y. S. Kivshar, and A. N. Poddubny, Inelastic Scattering of Photon Pairs in Qubit Arrays with Subradiant States, Phys. Rev. Lett. 123, 253601 (2019).

[29] J. Zhong, N. A. Olekhno, Y. Ke, A. V. Poshakinskiy, C. Lee, Y. S. Kivshar, and A. N. Poddubny, Photon-Mediated Localization in Two-Level Qubit Arrays, Phys. Rev. Lett. 124, 093604 (2020).

[30] J. Perczel, J. Borregaard, D. E. Chang, H. Pichler, S. F. Yelin, P. Zoller, and M. D. Lukin, Topological Quantum Optics in Two-Dimensional Atomic Arrays, Phys. Rev. Lett. 119, 023603 (2017).

[31] M. Bello, G. Platero, J. I. Cirac, and A. González-Tudela, Unconventional quantum optics in topological waveguide QED, Sc. Adv. 5, eaaw0297 (2019).
[32] C. A. Downing, T. J. Sturges, G. Weick, M. Stobińska, and L. Martín-Moreno, Topological Phases of Polaritons in a Cavity Waveguide, Phys. Rev. Lett. 123, 217401 (2019).

[33] S.-P. Yu, J. A. Muniz, C.-L. Hung, and H. J. Kimble, Twodimensional photonic crystals for engineering atom-light interactions, Proc. Natl. Acad. Sci. USA 116, 12743 (2019).

[34] M. A. Gorlach and A. N. Poddubny, Interaction-induced twophoton edge states in an extended Hubbard model realized in a cavity array, Phys. Rev. A 95, 033831 (2017).

[35] M. A. Gorlach and A. N. Poddubny, Topological edge states of bound photon pairs, Phys. Rev. A 95, 053866 (2017).

[36] Y. Ke, X. Qin, Y. S. Kivshar, and C. Lee, Multiparticle Wannier states and Thouless pumping of interacting bosons, Phys. Rev. A 95, 063630 (2017).

[37] X. Qin, F. Mei, Y. Ke, L. Zhang, and C. Lee, Topological magnon bound states in periodically modulated Heisenberg XXZ chains, Phys. Rev. B 96, 195134 (2017).

[38] X. Qin, F. Mei, Y. Ke, L. Zhang, and C. Lee, Topological invariant and cotranslational symmetry in strongly interacting multi-magnon systems, New J. Phys. 20, 013003 (2018).

[39] L. Lin, Y. Ke, and C. Lee, Interaction-induced topological bound states and Thouless pumping in a one-dimensional optical lattice, Phys. Rev. A 101, 023620 (2020).

[40] S. Aubry and G. André, Analyticity breaking and Anderson localization in incommensurate lattices, Ann. Isr. Phys. Soc. 3, 133 (1980).

[41] L.-J. Lang, X. Cai, and S. Chen, Edge States and Topological Phases in One-Dimensional Optical Superlattices, Phys. Rev. Lett. 108, 220401 (2012).

[42] A. N. Poddubny, Quasiflat band enabling subradiant twophoton bound states, Phys. Rev. A 101, 043845 (2020).

[43] M. Lyubarov and A. N. Poddubny, Exceptional points for photon pairs bound by nonlinear dissipation in cavity arrays, Opt. Lett. 43, 5917 (2018).

[44] Yu.-X. Zhang and K. Mølmer, Theory of Subradiant States of a One-Dimensional Two-Level Atom Chain, Phys. Rev. Lett. 122, 203605 (2019).

[45] F. K. Kunst, E. Edvardsson, J. C. Budich, and E. J. Bergholtz, Biorthogonal Bulk-Boundary Correspondence in Non-Hermitian Systems, Phys. Rev. Lett. 121, 026808 (2018).

[46] M. Di Liberto, A. Recati, I. Carusotto, and C. Menotti, Twobody physics in the Su-Schrieffer-Heeger model, Phys. Rev. A 94, 062704 (2016).

[47] J. Jin, X. Yin, L. Ni, M. Soljačić, B. Zhen, and C. Peng, Topologically enabled ultrahigh-Q guided resonances robust to out-of-plane scattering, Nature (London) 574, 501 (2019). 